\title{
Conceptual aircraft design exploration through functional approach
}

\begin{abstract}
In today's market, it is no longer sufficient to have better designs than the competition. In order to maximize the product's market potential, it has to be rapidly produced and made available to the market. To reduce their time-to-market period, manufacturers need to shorten their design and development process. It becomes vital that the design architecture solution is derived faster, which can be handful for complex products like an aircraft with the current geometrical-based approaches due to the plethora of physical alternatives to be considered. On the other hand, the search for design architecture solution from functional requirements is theoretically more effective because functional space is comparatively smaller than physical search space. This allows the design efforts to be more focused and this subsequently saves time, efforts and resources. With this notion, there is a driving motivation to adapt functional approaches into the conceptual product design process in order to exploit some of its advertised benefits. In this paper, an example case study of an aircraft conceptual development is presented to highlight possible advantages of approaching architecture solutions from the functional space.
\end{abstract}

Keyword: Aircraft system; Conceptual design; Functional approach 